\title{
Nothing to declare?
}

\author{
Declaring competing financial interests when publishing a methods paper has no negative \\ connotation, and proper disclosure is essential to maintain credibility and transparency.
}

\section{“I have nothing}

\section{to declare but}

\section{my genius."}

Oscar Wilde (1854-1900)
Medical publishing has recently come under fire from news media over reports that authors of some clinical studies had deliberately failed to disclose conflicting financial interests. Is the publication of methods papers immune to that affliction? Certainly not. Nowadays, new technologies and useful reagents stand a good chance of eventually being commercialized, thus attaching a considerable potential financial interest to the publication of a methods paper.

To promote transparency, Nature titles require authors of primary research papers, Reviews and Perspectives, to make one of three standardized public statements - choosing to declare competing financial interests, to declare they have none or to decline commenting. The motivation of this policy is to allow readers to form their own judgment, knowing any outside interest that has the potential to bias, deliberately or inadvertently, the authors' presentation of their work.

Nature Methods supports and implements this publishing policy, and we wish to stress that although making a false statement constitutes a serious offense, there should be no stigma associated with disclosure of financial interests in our pages. Nonetheless, editors occasionally come across reluctant authors who seem to consider that declaring competing financial interests means loosing credibility. Although difficult to pinpoint, the origin of such reluctance is probably rooted in the enduring myth of a divide between the virtuous, altruistic academic world and the commercially self-centered for-profit sector. But in this day and age, the border between for-profit and nonprofit sectors is being blurred by increasing pressures to patent academic work, exerted on investigators by their own employers, and by a growing involvement of industry in large-scale basic research projects.

In our experience, when authors of methods papers neglect to make a spontaneous disclosure, most often it is not a deliberate deception but a candid misunderstanding of what constitutes competing financial interests. A common example is when the 'Technology Office' at an academic institution has filed a patent application on behalf of the authors. Because the authors have not initiated the process and do not expect to receive the lion's share of any potential financial gain, they feel free of competing interests, but they may in fact benefit indirectly from a commercial success via funding from their institution or a small amount of royalties. Another common case is when authors employed by a company report on a method that is not currently a marketable product. Typically, a company owns the intellectual property on the work performed by its employees, and given the for-profit nature of the business, employment alone does constitute a competing interest worth reporting.

As for the potential for loss of credibility, there is no indication that a declaration of competing interest affects in any way the impact of a Nature Methods paper. In fact, the authors of $36 \%$ of papers published in Nature Methods in 2006 to date have declared competing financial interests. The potential for financial gain associated with the publication of a new method should not have a negative connotation. Arguably, for the types of papers we publish, commercialization of the content after publication can be viewed as a mark of success, or at least an indication that the new technique is in demand among other researchers.

Often, commercialization may also benefit the community by making a technology more widely available. This is especially true for instruments, devices and reagents, such as chemical probes or enzymes, which are difficult to produce consistently. For most researchers, buying quality-controlled reagents is a more cost-effective strategy than investing in their production.

Declaring competing financial interest at the time of publication can even be a positive thing for authors who, by filing a patent application, have envisioned a commercial prospect for their new method, but do not intend to lead the effort. Venture capitalists and their consultants have been known to screen the technical literature in their search for investment targets, and a patent application is an indication of an attractive, 'clean' situation in terms of intellectual property.

In sum, we encourage you, when in doubt, to err on the side of declaring all potential competing financial interests, the nature of which can always be qualified in the online statement accompanying the paper. In the current climate of media hype and public mistrust, it is particularly important to offer transparency. Although there is no negative connotation associated with such disclosure when publishing a new method, being exposed as someone who failed to disclose a competing financial interest is a clear invitation to suspicion. 